Journal for ImmunoTherapy of Cancer

\title{
Genetic ablation of adipocyte PD-L1 reduces tumor growth but accentuates obesity-associated inflammation
}

\author{
Bogang Wu (D , ${ }^{1}$ Huai-Chin Chiang, ${ }^{1}$ Xiujie Sun, ${ }^{1}$ Bin Yuan, ${ }^{1}$ Payal Mitra, ${ }^{1}$ \\ Yanfen $\mathrm{Hu},{ }^{1}$ Tyler J Curiel, ${ }^{2}$ Rong $\mathrm{Li}^{1}$
}

To cite: Wu B, Chiang H-C, Sun X, et al. Genetic ablation of adipocyte PD-L1 reduces tumor growth but accentuates obesityassociated inflammation. Journal for ImmunoTherapy of Cancer 2020;8:e000964. doi:10.1136/jitc-2020-000964

- Additional material is published online only. To view please visit the journal online (http://dx.doi.org/10.1136/jitc2020-000964).

Accepted 16 July 2020

\section{ABSTRACT}

The programmed death-ligand 1 (PD-L1)-dependent immune checkpoint attenuates host immunity and maintains self-tolerance. Imbalance between protective immunity and immunopathology due to altered PDL1 signaling can lead to autoimmunity or tumor immunosuppression. The role of the PD-L1-dependent checkpoint in non-immune system is less reported. We previously found that white adipocytes highly express PDL1. Here we show that adipocyte-specific PD-L1 knockout mice exhibit enhanced host anti-tumor immunity against mammary tumors and melanoma with low or no tumor PD-L1. However, adipocyte PD-L1 ablation in tumor-free mice also exacerbates diet-induced body weight gain, proinflammatory macrophage infiltration into adipose tissue, and insulin resistance. Low PD-L1 mRNA levels in human adipose tissue correlate with high body mass index and presence of type 2 diabetes. Therefore, our mouse genetic approach unequivocally demonstrates a cell-autonomous function of adipocyte PD-L1 in promoting tumor growth and inhibiting antitumor immunity. In addition, our work uncovers a previously unrecognized role of adipocyte PD-L1 in mitigating obesity-related inflammation and metabolic dysfunction.

\section{BACKGROUND}

Inhibitory checkpoints in biological systems limit the negative impact of uncontrolled cellular processes on biological integrity. As a salient example, the immune checkpoint molecule programmed death-ligand 1 (PDL1) is induced by host immunity-released inflammatory cytokines and in turn attenuates immune responses to infection, limits tissue damage, and maintains self-tolerance. When hijacked by tumors, PD-L1 expressed in tumor and certain immune cells dampens antitumor immunity via various mechanisms. ${ }^{23}$ Thus, tumor and immune cells have been the focus of extensive investigations of PD-L1 expression/function and their relevance to clinical efficacy of anticancer immunotherapies. ${ }^{45}$ In contrast, the potential influence of PD-L1 in other cell types on antitumor immunity is vastly underexplored. In addition, it is not clear whether the same principle of inhibitory checkpoint is applicable to non-immune systems in mitigation of homeostatic imbalance.

We recently identified white adipocytes as a previously unrecognized reservoir of PD-L1. ${ }^{6}$ We further showed that expression of mouse adipocyte PD-L1 mRNA and protein are markedly stimulated during adipogenesis. ${ }^{6}$ PD-L1 is also expressed in mouse brown adipose tissue (BAT). ${ }^{78}$ Despite these published findings, the functionality of adipocyte PD-L1 remains poorly understood. Here, we used a tissue-specific knockout (KO) mouse model to assess the role of adipocyte PD-L1 in antitumor immunity and adipose homeostasis.

\section{METHODS \\ Tumor study}

Mammary tumor E0771 was purchased from CH3 Biosystems (Cat: 940001). Melanoma B16 (Cat: CRL-6475) and lymphoma EL4 (Cat: TIB-39) were purchased from ATCC. Mammary tumor AT- ${ }^{9}$ was generated by Dr. Scott Abrams's lab at Roswell Park Comprehensive Cancer Center. Colorectal cancer MC-38-ova ${ }^{10}$ and luciferase reporter-positive ovarian cancer ID8agg ${ }^{11}$ cell lines were generated by Dr. Tyler Curiel's lab at University of Texas Health San Antonio. E0771 $\left(0.5 \times 10^{6}\right)$ and AT-3 $\left(0.2 \times 10^{6}\right)$ mammary tumor cell lines were transplanted orthotopically into the inguinal mammary fat pad of female mice. B16 melanoma $\left(0.5 \times 10^{6}\right)$, MC-38-ova colon cancer $\left(6.5 \times 10^{6}\right)$ and EL4 lymphoma cell lines $\left(2 \times 10^{6}\right)$ were transplanted subcutaneously into the back flank of male mice. $4 \times 10^{6}$ ID8agg cells were injected intraperitoneally. Tumor growth was monitored and immunophenotyping were performed as previously described. ${ }^{6}$ Survival of ovarian tumor-bearing mice was assessed by ascites formation, weight loss or distress. For assessment of melanoma metastasis, whole lung tissue was 
harvested from tumor-bearing mice after 16 days posttumor transplantation and snap-frozen for subsequent extraction of total RNA using an RNeasy mini kit (Cat: 74104, Qiagen). RT-qPCR was performed to quantify melanoma-specific transcripts in the lung. Primers used for qPCR $^{2}$ were as follow ${ }^{11}$ : $g p 100,5^{\prime}$-ACATTTCATCAC CAGCAGGGTGCC-3' and 5'-AACAAGTGGGTGCTGGCC-3'; Tyr, 5'-CTCTGGGCTTAGCAGTAGGC-3' and 5'-GCAAGCTGTGGTAGTCGTCT-3'; Trp-1, 5'-CCCC TAGCCTATATCTCCCTTTT-3' and 5'-TACCATCGTGGG GATAATGGC-3'; Trp-2, 5'-GTCCTCCACTCTTTTA CAGACG-3' and 5'-ATTCGGTTGTGACCAATGGGT; and $\beta$-Actin as the internal control, 5'-CAACGAGCGGTTCCGATG-3' and 5'-GCCACAGGATTCCATACCCA-3'. For assessment of metastatic ovarian tumors, whole liver tissue of ID8 tumor-bearing mice was harvested for genomic DNA extraction 47 days after tumor implantation. Primers used for q-PCR were as follows ${ }^{12}$ : luciferase reporter gene Luc2, 5'-CACCGTCGTATTCGTGAGCA-3' and 5'-AGTCGTACTCGTTGAAGCCG-3'; Ptger2 as the internal control, 5'-CCTGCTGCTTATCGTGGCTG-3' and 5'-GCCAGGAGAATGAGGTGGTC-3' .

\section{Flow cytometry}

Tumor tissue was cut into small pieces and passed through a $70 \mu \mathrm{m}$ cell strainer to obtain single cell suspension. Viability Ghost Dye Violet 510 (Cat: 13-0870, Tonbo Biosciences) was used to distinguish live and dead cells. Cells were then blocked with $\alpha \mathrm{CD} 16 / 32$ (Cat: 70-0161, Tonbo Biosciences) at $4^{\circ} \mathrm{C}$ for $15 \mathrm{~min}$ and stained with

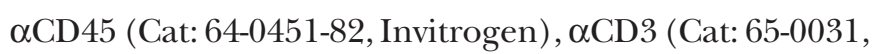
Tonbo Biosciences), $\alpha \mathrm{CD} 4$ (Cat: 78-0041-82, Invitrogen), $\alpha \mathrm{CD} 8$ (Cat: 557654, BD Pharmingen) at $4^{\circ} \mathrm{C}$ for $30 \mathrm{~min}$. For Ki67 staining, cells were permeabilized using FoxP3/ transcription factor staining kit (Cat: 00-5523-00, eBioscienceTM) and stained with $\alpha$ Ki67 (Cat: 48-5698-82, Invitrogen). For cytokine staining, cells were incubated with $\alpha \mathrm{CD} 3 / \mathrm{CD} 28$ (Cat: 11452D, ThermoFisher) for overnight at $37^{\circ} \mathrm{C}$ and treated with an activation cocktail with $\mathrm{BD}$ GolgiPlug (Cat: 550583, BD Biosciences) for 5 hours at $37^{\circ} \mathrm{C}$. Cells were permeabilized using BD Cytofix/Cytoperm kit (Cat: 554714, BD Biosciences) and stained with $\alpha$ Perforin (Cat: 11-9392-82, Invitrogen) for $30 \mathrm{~min}$ at $4^{\circ} \mathrm{C}$. Relevant controls included single color staining, isotype controls or fluorescence minus one. For PD-L1 quantification in various cell lines, cells were incubated with $5 \mathrm{ng}$ / $\mathrm{mL}$ recombinant interferon $\gamma(\mathrm{IFN} \gamma)$ overnight at $37^{\circ} \mathrm{C}$ and stained with a viability Ghost Dye Violet 510 (Cat: 13-0870, Tonbo Biosciences) and $\alpha$ PD-L1 (Cat: 46-598282, Invitrogen). Data acquisition was conducted using BD FACSCelesta and analysis was done using FlowJo software (BD Bioscience) or BD FACSDiva.

\section{RNA sequencing}

Tumor and fat tissue RNA extraction was performed using RNeasy Mini Kit (Cat: 74104, Qiagen) and RNeasy lipid tissue mini kit (Cat: 74804, Qiagen), respectively. For RNA sequencing, about $500 \mathrm{ng}$ total RNA from each sample was used for downstream library preparation
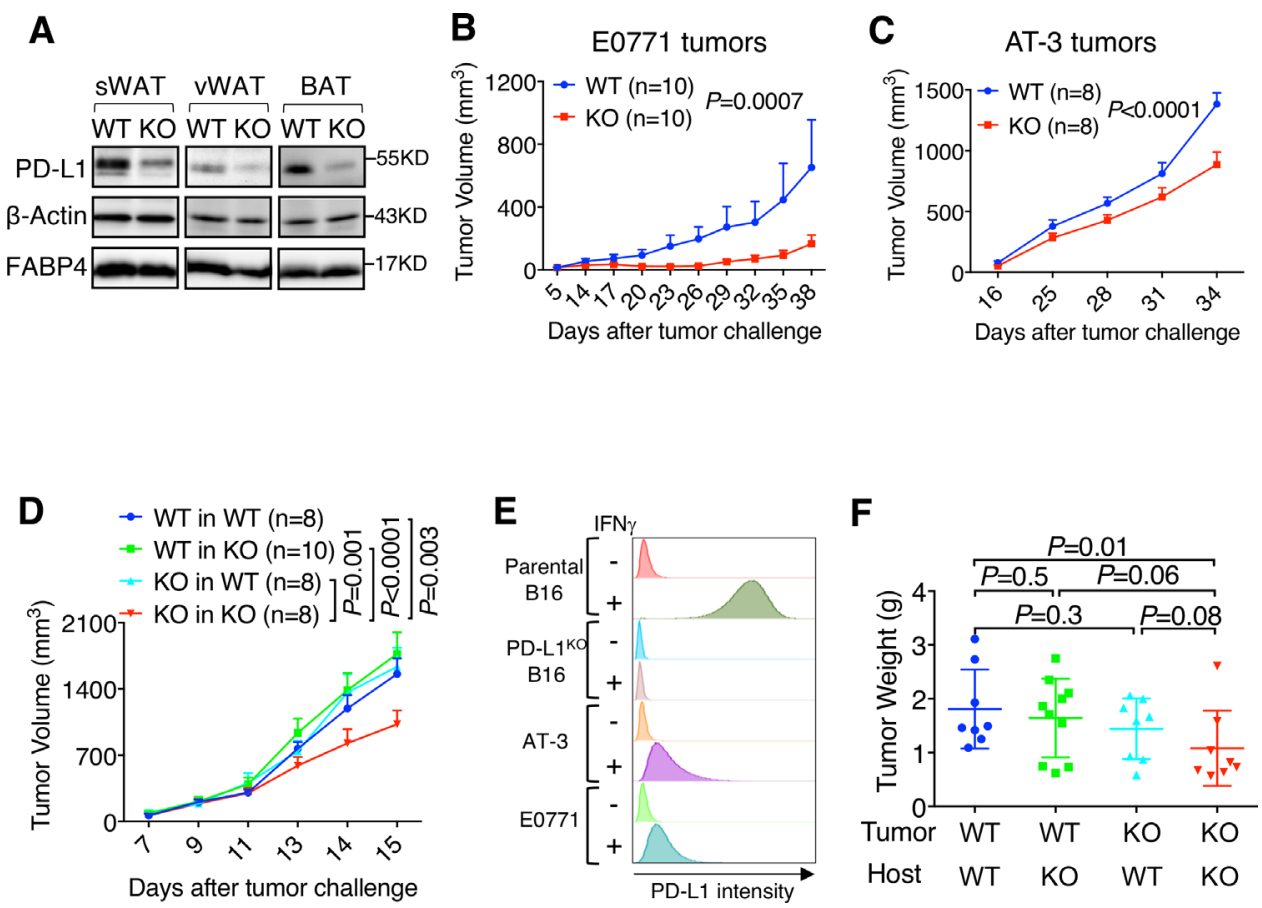

Figure 1 Adipocyte programmed death-ligand 1 (PD-L1) ablation reduces tumor growth. (A) PD-L1 protein levels in subcutaneous white adipose tissue (sWAT), visceral white adipose tissue (vWAT) and brown adipose tissue (BAT). (B) E0771 and (C) AT-3 mammary tumor growth in wild-type (WT)/knockout (KO) mice. (D) Growth of parental and PD-L1 KO B16 melanoma in WT/KO mice. (E) Flow cytometry quantification of PD-L1 in tumor cell lines, \pm interferon $\gamma$ (IFN $\gamma$ ) treatment. (F) Tumor weight measurement. 
according to the Illumina TruSeq stranded mRNA-seq guidance. Briefly, poly-T oligo-attached magnetic beads were used to enrich poly A-containing mRNA. Random primers and reverse transcriptase were then used to convert enriched mRNA to first-strand cDNA. DNA polymerase I and RNase $\mathrm{H}$ were then used to synthesize the second-strand cDNA. PCR methods were used to generate final RNA-seq libraries. RNA-seq libraries were quantified and pooled for cBot amplification. $50 \mathrm{bp}$ single read sequencing was carried out with Illumina HiSeq 3000 platform. After sequencing, fastq files was generated using demultiplexing with Bcl2fastq2.

\section{Metabolism analysis}

Mice were fed a high-fat diet (HFD; $60 \mathrm{kcal} \%$ fat) or low-fat diet (LFD; $10 \mathrm{kcal} \%$ fat) starting at 6 weeks after birth. Glucose and insulin tolerance were assessed by intraperitoneal injection of $2 \mathrm{~g} / \mathrm{kg}$ glucose and 1.8 $\mathrm{mU} / \mathrm{g}$ human insulin, respectively. For macrophage characterization, visceral white adipose tissue (vWAT) was digested into single-cell suspensions and examined by flow cytometry. F4/80 immunohistochemistry and liver H\&E staining were carried out at VitroVivo Biotech, LLC. For human sample analysis, cancer-free breast tissue was procured per an Institutional Review
Board protocol. Fresh breast tissue was digested and sorted for $\mathrm{Lin}^{-}$cells before RNA extraction.

\section{Statistics}

Mean differences between two groups were compared using unpaired Student's t-test. Mean differences from multiple groups were compared by one-way analysis of variance. Survival analysis was done by Log-rank (Mantel-Cox) test. RNA-seq analysis was performed with Gene Ontology, gene set enrichment analysis (GSEA) analysis and DESeq using R. Image analysis was performed using Image J. Statistics were performed by GraphPad Prism. $\mathrm{P}<0.05$ was considered significant.

\section{RESULTS}

Adipocyte-specific PD-L1 ablation significantly enhances host antitumor immunity and slows tumor growth

To determine the biological function of adipocyte PD-L1, we established an adipocyte-specific PD-L1 KO mouse model (Adiponectin-Cre, PD-L $f^{f / f}$ in C57BL/6), which reduced PD-L1 protein in subcutaneous and visceral white adipose tissue (sWAT, vWAT) and BAT (figure 1A) without changing PD-L1 expression in immune cells from spleen, lymph nodes, or thymus (online supplementary
A

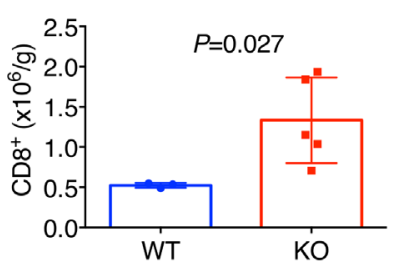

D

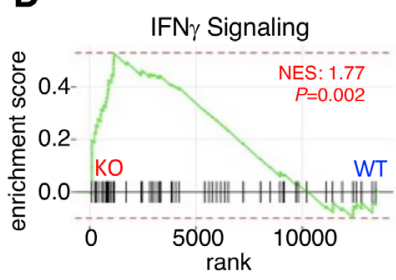

G

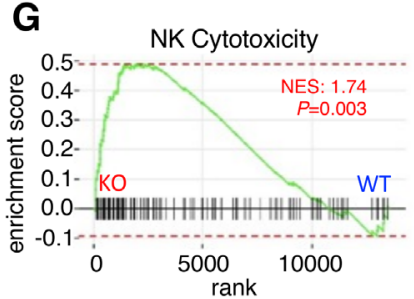

B

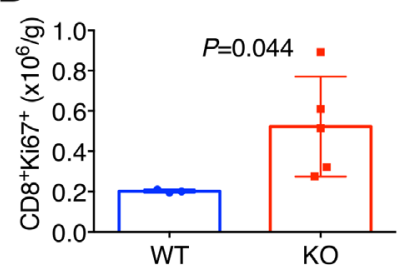

E

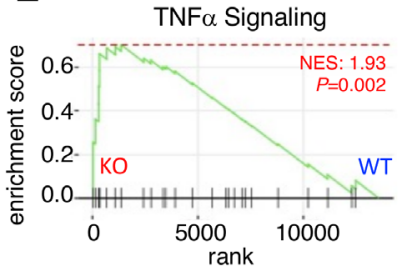

H

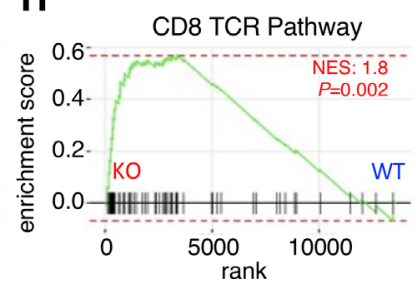

C

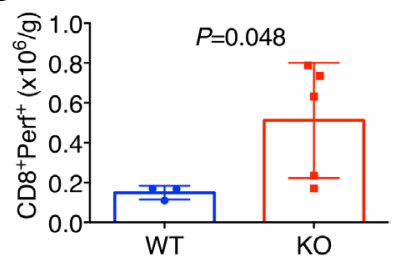

F
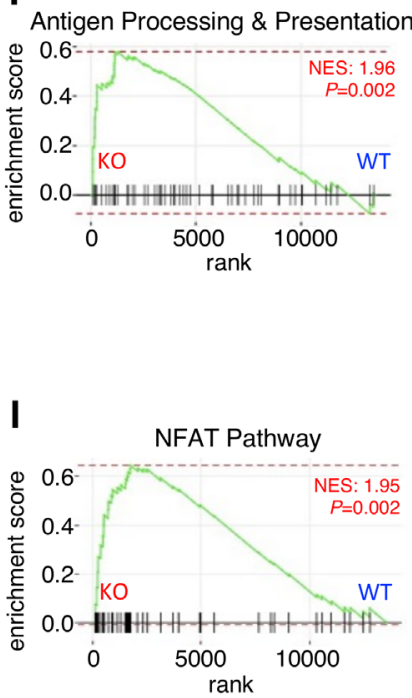

Figure 2 Adipocyte programmed death-ligand 1 (PD-L1) ablation enhances antitumor immunity. (A) Flow cytometry of E0771 tumor-infiltrating $\mathrm{CD}^{+}$, (B) $\mathrm{CD}^{+} \mathrm{Ki}^{+} 7^{+}$and (C) $\mathrm{CD}^{+} \mathrm{Perf}^{+}$cell content. (D-F) Gene sets enrichment analysis for E0771 tumors from wild-type (WT)/knockout (KO) hosts. IFN, interferon; NES, normalized enrichment score; TNF, tumor necrosis factor; NK, Natural killer; TCR, T cell receptor; NFAT: Nuclear factor of activated T-cells. 
figure S1A-C). Adipocyte PD-L1 KO mice did not exhibit any gross developmental defects or appreciable body weight differences versus their wild-type (WT) littermates when fed normal chow (see below). However, syngeneic mammary tumors (E0771 and AT-3) grew significantly slower in KO versus WT hosts (figure 1B,C), indicating that adipocyte PD-L1 has a tumor-promoting function. A similar observation was made in syngeneic tumor models for primary and metastatic ovarian cancer (ID8agg, online supplementary figure S2A-B) and primary colorectal cancer (MC38, online supplementary figure S2C), but not for lymphoma (EL4, online supplementary figure S2D) or melanoma (B16, figure 1D), suggesting that additional tumor and/or stromal factors influence the tumor-promoting effect of adipocyte PD-L1.

In considering tumor-intrinsic factors that could affect the functional outcome of adipocyte PD-L1, we noted that PD-L1 expression in B16 melanoma and EL4 lymphoma is significantly higher than PD-L1 in those tumors that exhibit differential growth in WT and adipocyte PD-L1 $\mathrm{KO}$ hosts (figure 1E, online supplementary figure S2E). We therefore asked whether high tumor PD-L1 expression could mask the adipocyte PD-L1 effect on tumor growth. To test this possibility, we challenged WT mice with PD-L1 KO B16 cells and found that they grew similar to control B16 cells in WT hosts (figure 1D). By contrast, PD-L1 KO B16 melanoma grew significantly slower in PD-L1 KO versus WT hosts (figure 1D,F), an observation made in both primary and metastatic tumor sites (online supplementary figure S2F-G). These data suggest that the adipocyte PD-L1 effect on tumor growth at least partly depends on tumor PD-L1. This is consistent with previous reports concerning the relative contribution of tumor and stromal PD-L1 to tumor progression. ${ }^{13}$

Analysis of mammary tumor-infiltrating lymphocytes by flow cytometry showed an increased total (figure 2A, online supplementary figure S3A), proliferating (figure 2B, online supplementary figure $\mathrm{S} 3 \mathrm{~B}$ ), and perforin-expressing $\mathrm{CD}^{+} \mathrm{T}$ cell content in $\mathrm{KO}$ hosts (figure 2C, online supplementary figure S3C), suggesting that adipocyte PD-L1 dampens host antitumor immunity. This is also consistent with our previous in vitro finding that white adipocyte PD-L1 antagonizes $\mathrm{CD}^{+}$cytotoxic $\mathrm{T}$ cell functions. ${ }^{6}$ In further support, gene ontology analysis of RNA-seq data shows that pathways related to antitumor immune responses including cytokine
A

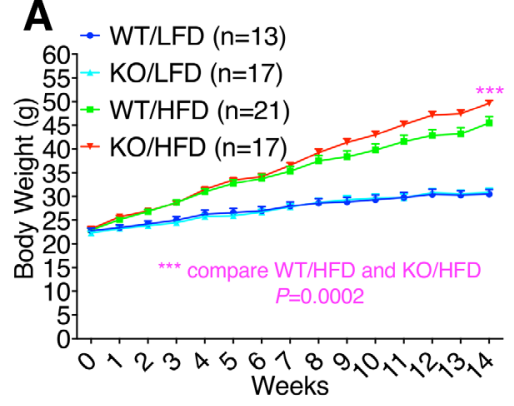

B

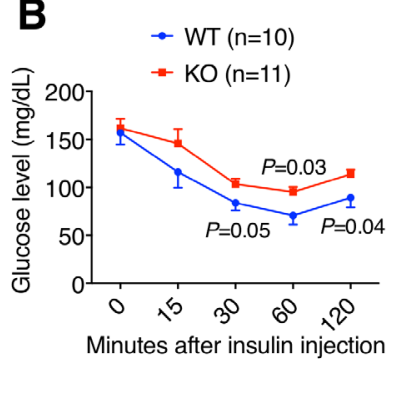

C

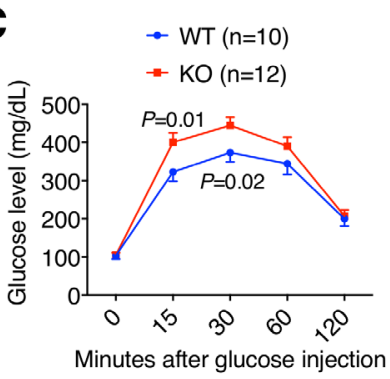

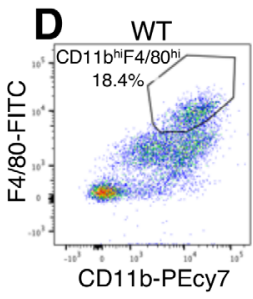
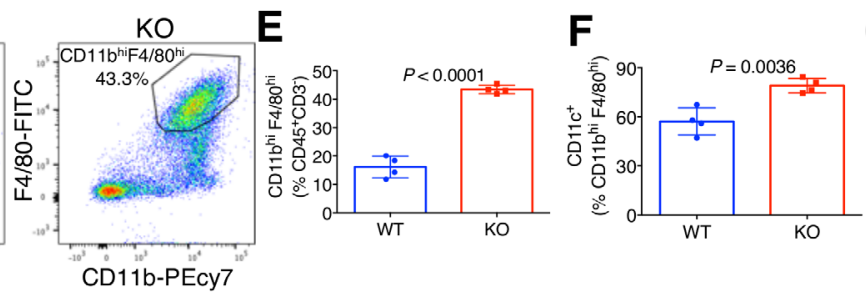

G
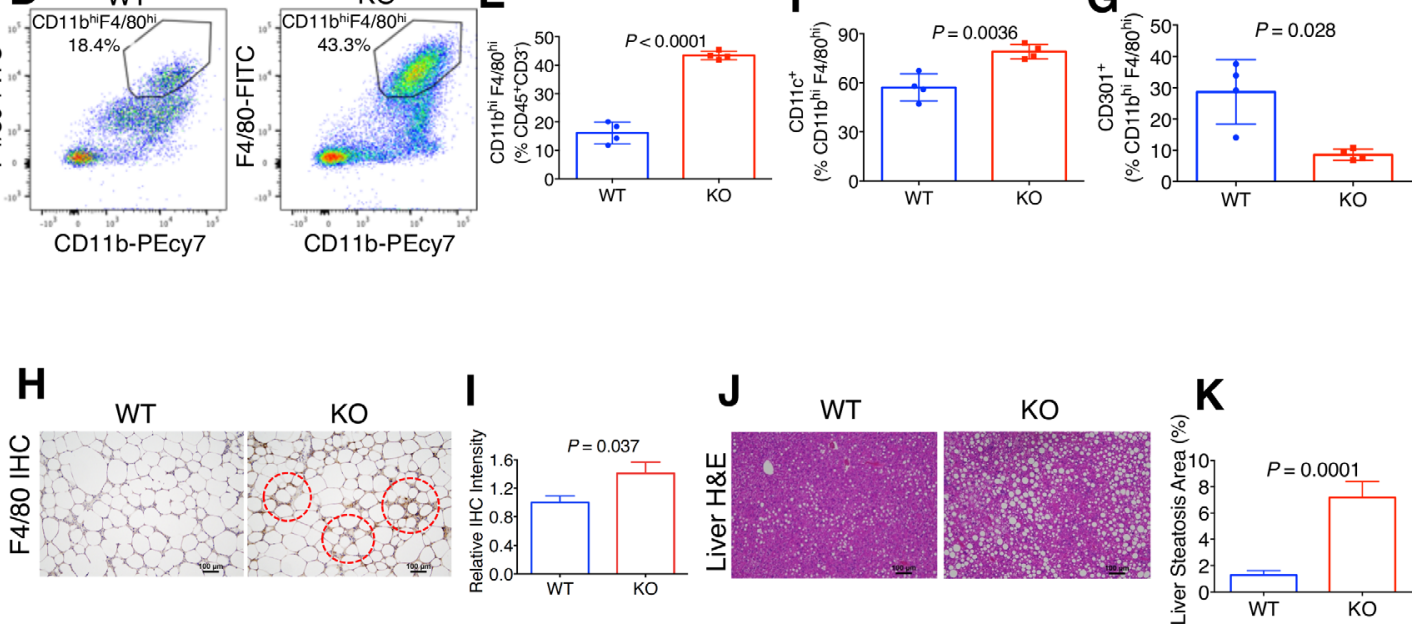

Figure 3 Programmed death-ligand 1 (PD-L1) deletion exacerbates obesity-associated inflammation and metabolic distress. (A) Body weight of wild-type (WT)/knockout (KO) mice given low-fat diet (LFD) or high-fat diet (HFD). (B) Insulin tolerance in obese WT/KO mice. (C) Glucose tolerance. (D) Representative flow cytometry data for CD11 b ${ }^{\text {hi }}$ F $/ 80^{\text {hi }}$ macrophage population in obese WT/KO visceral white adipose tissue (vWAT). (E) Macrophage prevalence in obese WT/KO vWAT. (F) Prevalence of $\mathrm{CD} 11 \mathrm{c}^{+}$and $(\mathrm{G}) \mathrm{CD} 01^{+}$percentage of total macrophages. (H) Representative F4/80 immunohistochemistry (IHC). (I) Quantification of relative F4/80 IHC signals. WT level is set as "1." (J) Representative liver H\&E staining. (K) Quantification of liver steatosis area as percentage of the entire liver tissue. 
production $(\mathrm{p}=1.00 \mathrm{E}-07)$ and granzyme-mediated apoptotic signaling pathway $(\mathrm{p}=4.00 \mathrm{E}-06)$ were substantially upregulated in tumors from the KO hosts versus WT. In addition, GSEA indicates that, compared with WT, tumors from the KO hosts had significantly enriched gene sets in IFN $\gamma$ signaling (figure 2D), tumor necrosis factor $\alpha$ signaling (figure 2E), antigen processing and presentation (figure 2F), natural killer cell cytotoxicity (figure $2 \mathrm{G}$ ), $\mathrm{CD}^{+} \mathrm{T}$ cell receptor signals (figure $2 \mathrm{H}$ ), and NFAT signals (figure 2I). To extend our mouse tumor study to human clinical samples, we analyzed a published database comparing transcriptomes of normal and breast cancer-associated stromal tissue and found significantly higher PD-L1 mRNA expression in the stroma of invasive breast carcinoma versus that from normal breast tissue $(\mathrm{p}=6.08 \mathrm{E}-5) .{ }^{14}$ Taken together, these data strongly support the notion that adipocyte PD-L1 plays a previously unrecognized, cell-autonomous role in curbing antitumor immunity and promoting tumor progression.

\section{PD-L1 ablation exacerbates obesity-associated inflammation and metabolic distress}

Given the central role of white adipocytes in obesity, we asked whether adipocyte PD-L1 affected obesity and associated metabolic stress in tumor-free mice. Because estrogens are known to protect female mice from diet-induced obesity and metabolic dysfunction, ${ }^{15-17}$ we fed male WT/ KO mice either a low-fat diet (LFD 10\% kcal\% fat) or high fat diet (HFD $60 \% \mathrm{kcal} \%$ fat). Male WT and KO mice fed LFD exhibited equivalent weight gain (figure 3A). In contrast, $\mathrm{KO}$ male mice fed HFD gained substantially more weight than obese WT (figure 3A). Insulin tolerance and glucose tolerance were impaired in obese $\mathrm{KO}$ versus their WT counterparts (figure 3B,C). Thus, adipocyte PD-L1 appears to mitigate obesity and aspects of metabolic syndrome.

Flow cytometry-based analysis indicates a marked increase in vWAT macrophage prevalence in obese KO versus WT mice (figure 3D-E). Furthermore, KO vWAT had a higher prevalence of macrophages expressing the proinflammatory marker CD11c (figure 3F) and lower prevalence expressed the anti-inflammatory marker CD301 (figure 3G). These immunophenotyping data are consistent with previously reported changes of macrophages markers under obese condition. ${ }^{18}$ Crown-like structure, an established marker for obesity-associated inflammation and adipocyte death, was more prevalent in obese $\mathrm{KO}$ versus WT adipose tissue (figure $3 \mathrm{H}-\mathrm{I}$ ). Furthermore, hepatic steatosis, indicative of metabolic dysfunction, was more pronounced in obese KO than WT (figure 3J-K).
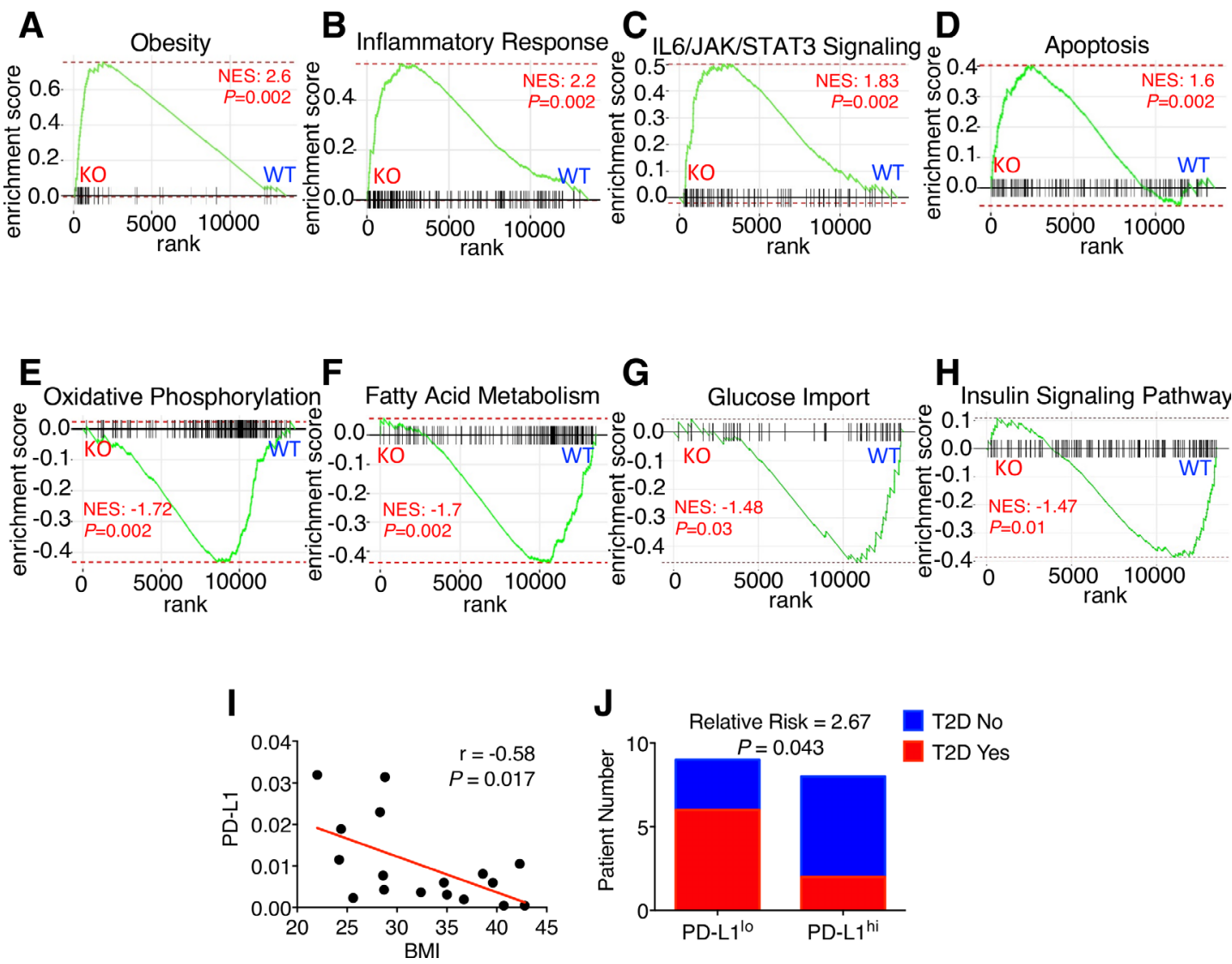

Figure 4 Low adipocyte programmed death-ligand 1 (PD-L1) correlates with fat tissue inflammation and metabolic disorder. (A-H) Gene set enrichment analysis for obese wild-type (WT)/knockout (KO) visceral white adipose tissue (vWAT). (I) Correlation between human adipose PD-L1 mRNA and body mass index (BMI). (J) Type 2 diabetes (T2D) incidence in PD-L $1^{\text {lo }}$ and PD-L1 ${ }^{\text {hi }}$ groups. IL, interleukin. 
To further corroborate the cell-based findings, we conducted RNA-seq using adipose tissue samples of obese WT and KO mice. Bioinformatic analysis indicates enrichment of genes associated with obesity (figure 4A), inflammation (figure 4B), interleukin-6/JAK/STAT3 signaling (figure 4C), and apoptosis (figure 4D) in $\mathrm{KO}$ versus WT vWAT. In addition, genes related to cell migration $(\mathrm{p}=4.70 \mathrm{E}-30)$ and immune response $(\mathrm{p}=2.30 \mathrm{E}-30)$ were significantly elevated in the obese $\mathrm{KO}$ group. Remarkably, mRNA levels of Itgax, which encodes the proinflammatory macrophage marker CD11c (figure 3F), was approximately eightfold higher in obese $\mathrm{KO}$ than WT $(p=3.03 E-25)$. Conversely, gene sets enriched in obese WT adipose samples versus obese $\mathrm{KO}$ include oxidative phosphorylation (figure 4E), fatty acid metabolism (figure $4 \mathrm{~F}$ ), glucose import (figure 4G) and insulin signaling pathway (figure $4 \mathrm{H}$ ), further supporting our glucose tolerance and insulin tolerance data (figure 4B-C). Collectively, our results strongly support the notion that adipocyte PD-L1 attenuates obesity-associated inflammation and metabolic stress.

To assess human relevance, we analyzed PD-L1 mRNA in breast adipose tissue from women undergoing elective reduction mammoplasties $(\mathrm{n}=17)$. We found a significant inverse correlation between adipocyte PD-L1 expression and body mass index (figure 4I). Individuals with low adipocyte PD-L1 expression were more likely to have type 2 diabetes (figure 4J); however, the small cohort size precludes further stratification by BMI. These findings are consistent with the concept that low adipocyte PD-L1 expression is associated with metabolic dysfunction, but much additional work is needed.

\section{DISCUSSION}

Using a PPAR $\gamma$ antagonist, our previously published study has shown that preferential reduction of adipocyte PD-L1 expression boosts antitumor immunity and inhibits tumor growth. ${ }^{6}$ Together with the mouse genetic approach in the current study, our work unequivocally identifies white adipocytes as a previously unrecognized, functionally important source of PD-L1 signals. Notably, our data define two opposing health-related outcomes of adipocyte PD-L1: tumor growth promotion that is likely through dampening antitumor immunity, and mitigation of obesityrelated pathologies including chronic inflammation and insulin resistance. Accumulating evidence indicates that caloric intake can trigger either metabolically healthy or unhealthy expansion of white adipose tissue. ${ }^{19}{ }^{20}$ Healthy WAT expansion involves increased adipogenesis ("hyperplastic"). On the other hand, unhealthy adipose expansion is associated with increased hypertrophy and decreased adipogenesis ("hypertrophic"), which ultimately leads to chronic low-grade inflammation and metabolic dysfunction such as insulin resistance. Our findings support the notion that adipogenesis-associated increase in adipocyte PD-L1 expression helps maintain adipose homeostasis and mitigate chronic inflammation during healthy expansion of adipose tissue. Conversely, diminished adipocyte PD-L1 levels as shown in our mouse genetic model and obese humans likely aggravate adipose infiltration of inflammatory macrophages and obesity-related metabolic disorders.

Obesity is associated with increased cancer risk and poor prognosis for certain cancer types. ${ }^{21}{ }^{22}$ The molecular contribution of adipose tissue to cancer is multifactorial. While numerous adipose tissue-derived factors have been reported to promote both obesity and cancer progression, our current finding of adipocyte PD-L1 provides a salient example of how a previously underappreciated, adipocyteintrinsic molecule can influence obesity and tumor growth in an opposing manner. While the underlying mechanism(s) of adipocyte PD-L1 action remains to be thoroughly investigated, the fact that depletion of adipocyte PD-L1 results in two divergent health-related consequences highlights the complex and multifaceted nature of adipose tissue in pathogenesis. In discerning the molecular basis of obesity-related cancer risk, it is therefore important to distinguish the overall impact of adiposity on cancer from the action of individual adipose tissue-derived factors.

Based on the data presented in this study, we propose the concept of a PD-L1-mediated adipose checkpoint that modulates obesity-associated chronic inflammation. More specifically, white adipocyte PD-L1 acts as an adipose checkpoint that attenuates obesity-associated macrophage recruitment into adipose tissue, chronic inflammation, and metabolic dysfunction. Mechanistically, adipocyte PD-L1 could execute adipose checkpoint effects by partnering with PD-1 from other cell types in adipose tissue and/or through regulating adipocyte-secreted cytokines. Of note, macrophages express PD-1, with higher expression in antiinflammatory M2-like versus proinflammatory M1-like subtype. ${ }^{23}$ In particular, PD-1 expressed in tumor-associated macrophages dampens phagocytosis and antitumor immunity. ${ }^{23}{ }^{24}$ Furthermore, PD-1 is known to induce polarization to the M2 phenotype while suppressing the M1 phenotype. $^{23} 25$ Thus, communication between macrophage PD-1 and adipocyte PD-L1 in adipose tissue could curtail macrophage-associated chronic inflammation and thus ameliorate homeostatic imbalance.

Acknowledgements The authors thank the UTHSCSA deep sequencing core for technical assistance.

Contributors RL and TC conceived and supervised the project. RL, TC, and BW designed the experiments. BW, H-CC, XS, BY, and PM performed the experiments. $\mathrm{RL}, \mathrm{TC}, \mathrm{YH}$, and BW analyzed the data. RL and BW wrote the manuscript.

Funding The work was supported by grants to RL (NIH CA206529 and DK115219); YH (NIH CA212674 DOD W81XWH-17-1-0007); TC (NIH CA205965 and CA231325) and the Owens and Barker Foundations and the Skinner Endowment.

Competing interests None declared.

Patient consent for publication Not required.

Ethics approval All animal-related procedures were approved by the Institutional Animal Care and Use Committee. For human sample analysis, cancer-free breast tissue was procured according to an Institutional Review Board-approved protocol.

Provenance and peer review Not commissioned; externally peer reviewed.

Data availability statement Data are available in a public, open access repository. All relevant data are included in the manuscript. The RNA-seq dataset has been deposited in NCBI GE0 database (accession number: GSE148149). 
Open access This is an open access article distributed in accordance with the Creative Commons Attribution Non Commercial (CC BY-NC 4.0) license, which permits others to distribute, remix, adapt, build upon this work non-commercially, and license their derivative works on different terms, provided the original work is properly cited, appropriate credit is given, any changes made indicated, and the use is non-commercial. See http://creativecommons.org/licenses/by-nc/4.0/.

ORCID iD

Bogang Wu http://orcid.org/0000-0003-1654-198X

\section{REFERENCES}

1 Sun C, Mezzadra R, Schumacher TN. Regulation and function of the PD-L1 checkpoint. Immunity 2018;48:434-52.

2 Baumeister SH, Freeman GJ, Dranoff G, et al. Coinhibitory pathways in immunotherapy for cancer. Annu Rev Immunol 2016;34:539-73.

3 Cha J-H, Chan L-C, Li C-W, et al. Mechanisms controlling PD-L1 expression in cancer. Mol Cell 2019;76:359-70.

4 Sanmamed MF, Chen L. A paradigm shift in cancer immunotherapy: from enhancement to normalization. Cell 2018;175:313-26.

5 Wei SC, Duffy CR, Allison JP. Fundamental mechanisms of immune checkpoint blockade therapy. Cancer Discov 2018;8:1069-86.

6 Wu B, Sun X, Gupta HB, et al. Adipose PD-L1 modulates PD-1/PDL1 checkpoint blockade immunotherapy efficacy in breast cancer. Oncoimmunology 2018;7:e1500107.

7 Hettich M, Braun F, Bartholomä MD, et al. High-Resolution PET imaging with therapeutic antibody-based PD-1/PD-L1 checkpoint tracers. Theranostics 2016;6:1629-40.

8 Ingram JR, Dougan M, Rashidian M, et al. Pd-L1 is an activationindependent marker of brown adipocytes. Nat Commun 2017;8:647.

9 Stewart TJ, Abrams SI. Altered immune function during long-term host-tumor interactions can be modulated to retard autochthonous neoplastic growth. J Immunol 2007;179:2851-9.

10 Hurez V, Daniel BJ, Sun L, et al. Mitigating age-related immune dysfunction heightens the efficacy of tumor immunotherapy in aged mice. Cancer Res 2012;72:2089-99.

11 Clark CA, Gupta HB, Sareddy G, et al. Tumor-Intrinsic PD-L1 signals regulate cell growth, pathogenesis, and autophagy in ovarian cancer and melanoma. Cancer Res 2016;76:6964-74.
12 Deng W, McLaughlin SL, Klinke DJ. Quantifying spontaneous metastasis in a syngeneic mouse melanoma model using real time PCR. Analyst 2017;142:2945-53.

13 Lau J, Cheung J, Navarro A, et al. Tumour and host cell PD-L1 is required to mediate suppression of anti-tumour immunity in mice. Nat Commun 2017;8:14572.

14 Finak G, Bertos N, Pepin F, et al. Stromal gene expression predicts clinical outcome in breast cancer. Nat Med 2008;14:518-27.

15 Pettersson US, Waldén TB, Carlsson P-O, et al. Female mice are protected against high-fat diet induced metabolic syndrome and increase the regulatory $\mathrm{T}$ cell population in adipose tissue. PLoS One 2012; 7:e46057.

16 Stubbins RE, Najjar K, Holcomb VB, et al. Oestrogen alters adipocyte biology and protects female mice from adipocyte inflammation and insulin resistance. Diabetes Obes Metab 2012;14:58-66.

17 Stubbins RE, Holcomb VB, Hong J, et al. Estrogen modulates abdominal adiposity and protects female mice from obesity and impaired glucose tolerance. Eur J Nutr 2012;51:861-70.

18 Cho KW, Morris DL, Lumeng CN. Flow cytometry analyses of adipose tissue macrophages. Methods Enzymol: Elsevier, 2014: 297-314.

19 Tandon P, Wafer R, Minchin JEN. Adipose morphology and metabolic disease. J Exp Biol 2018;221:jeb164970.

20 Longo M, Zatterale F, Naderi J, et al. Adipose tissue dysfunction as determinant of obesity-associated metabolic complications. Int $\mathrm{J} \mathrm{Mol}$ Sci 2019;20:2358

21 lyengar NM, Gucalp A, Dannenberg AJ, et al. Obesity and cancer mechanisms: tumor microenvironment and inflammation. J Clin Oncol 2016;34:4270-6.

22 Olson OC, Quail DF, Joyce JA. Obesity and the tumor microenvironment. Science 2017;358:1130-1.

23 Gordon SR, Maute RL, Dulken BW, et al. Pd-1 expression by tumourassociated macrophages inhibits phagocytosis and tumour immunity. Nature 2017;545:495-9.

24 Strauss L, Mahmoud MAA, Weaver JD, et al. Targeted deletion of PD-1 in myeloid cells induces antitumor immunity. Sci Immunol 2020;5:eaay 1863.

25 Lu D, Ni Z, Liu X, et al. Beyond T cells: understanding the role of PD-1/PD-L1 in tumor-associated macrophages. J Immunol Res 2019;2019:1-7. 